\title{
SOI MEMS PROCESS INSENSITIVE TO SACRIFICIAL OXIDE ETCH INDUCED SUBSTRATE ANCHOR PERIMETER VARIATION
}

\author{
G. J. O'Brien ${ }^{1}$, D. J. Monk ${ }^{2}$ and K. Najafi ${ }^{3}$ \\ ${ }^{1}$ Arizona State University, Department of Electrical Engineering, Tempe, AZ 85287 \\ ${ }^{2}$ Freescale Semiconductor, Sensor and Analog Products Division, Tempe, AZ 85284 \\ ${ }^{3}$ The University of Michigan, Department of Electrical Engineering, Ann Arbor, MI 48109
}

\begin{abstract}
A major challenge of SOI based MEMS transducer design and fabrication has been to provide reliable substrate anchors insensitive to timed sacrificial oxide etch induced perimeter variation. Typically, SOI substrate anchors are designed quite large to account for anchor perimeter variation due to the lack of an etch stop mechanism. This paper describes the use of silicon nitride, as a sacrificial oxide etch stop film, patterned and deposited via a novel trench refill process. Additionally, this trench refill process can be further improved using polysilicon to provide a low electrical resistance substrate contact between the SOI and silicon handle wafer. A process combining both the silicon nitride substrate anchor perimeter etch stop and polysilicon substrate electrical contact is provided with device characterization results.
\end{abstract}

\section{INTRODUCTION}

SOI mechanical substrate anchors are typically fabricated by utilizing the buried oxide layer as a readily available electrical insulator and easily etched sacrificial material via hydrofluoric (HF) acid. A process cross section of a typical SOI timed HF etch defined oxide substrate anchor is shown in Figure 1 where $x$ represents the desired lateral undercut dimension.

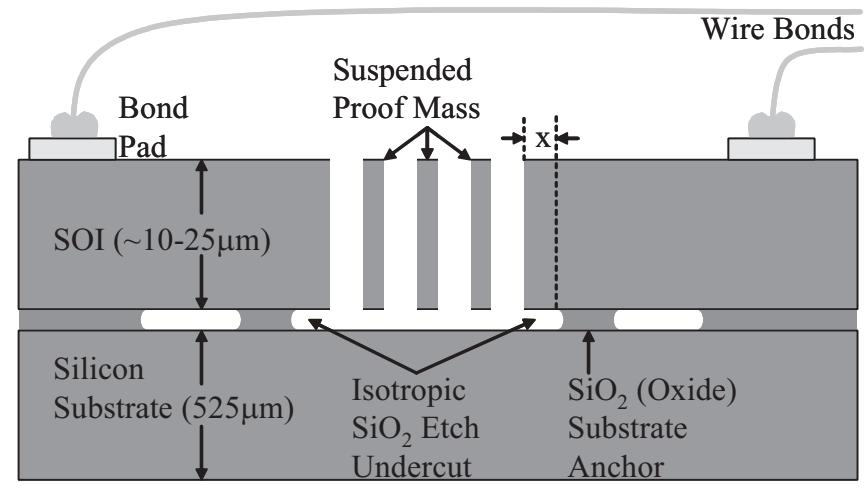

Figure 1. Cross section of typical SOI timed HF etch lateral undercut defined oxide substrate anchors.

This lateral undercut dimension $(x)$ is a strong function of time and initial-final HF concentration. A non-exhaustive list of variables contributing to lateral undercut etch variation include trench width, trench depth, buried oxide thickness, and HF concentration as a function of time, temperature, etc. Several of these variables are difficult to control when batch processing high volumes of silicon wafers in a manufacturing environment. MEMS SOI sensor designers typically make the anchors much larger than desirable in order to guarantee device reliability as a function of lateral etch undercut variability as observed across the device, wafer, and lot over time. This increases the design's required area consequently resulting in higher production cost per die. Therefore, an etch-stop based mechanical substrate anchor which allows the designer to use a smaller anchor area insensitive to timed HF lateral undercut variability based on trench refill processing [1] is highly desirable.

\section{SOI DEVICE FABRICATION}

A process cross section of silicon nitride trench refill encapsulated oxide substrate anchors insensitive to HF timed etch variation $[2,3]$ is shown in Figure 2.

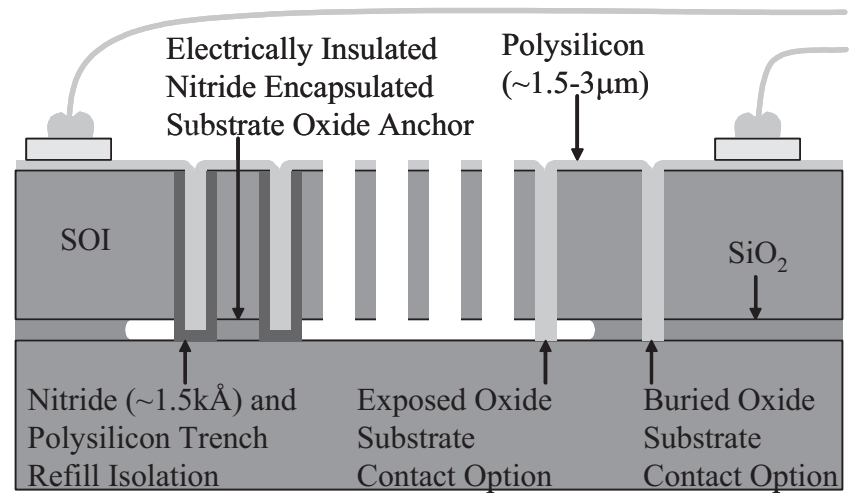

Figure 2. Cross section of silicon nitride encapsulated buried oxide substrate anchors insensitive to HF timed etch variation.

The low pressure vapor phase deposition (LPCVD) silicon nitride film serves as an electrical insulator, HF etch stop, and diffusion barrier. An LPCVD polysilcon flim is used to back fill the trench due to its high degree of as deposited conformality. The nitride film serves as a diffusion barrier between the degenerately doped adjacent SOI and intrinsic LPCVD polysilicon film. A capacitive angular acceleration sensor [4] fabricated in SOI using this process flow [5] is shown in Figure 3.

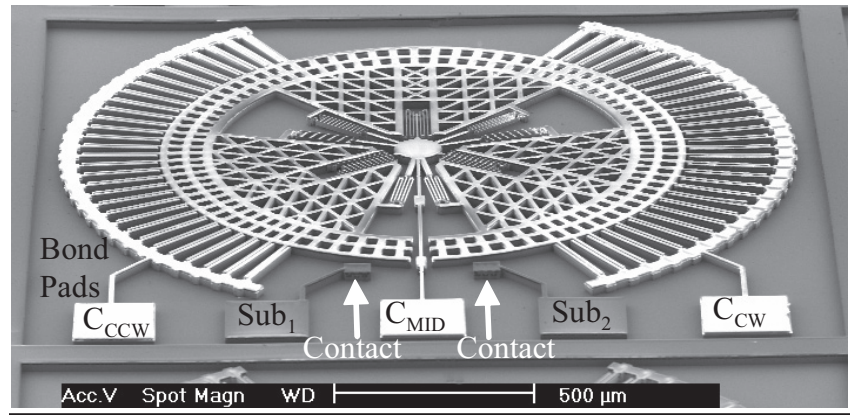

Figure 3. Angular accelerometer fabricated $w /$ substrate contacts.

The silicon nitride film functions as an electrical insulator and HF etch stop surrounding the encapsulated buried oxide anchor perimeter as shown in Figure 4. This silicon nitride encapsulated buried oxide anchor represents a substrate anchor 
with the bond strength between the SOI and silicon handle wafer approaching the fracture strength of single crystal silicon [6].

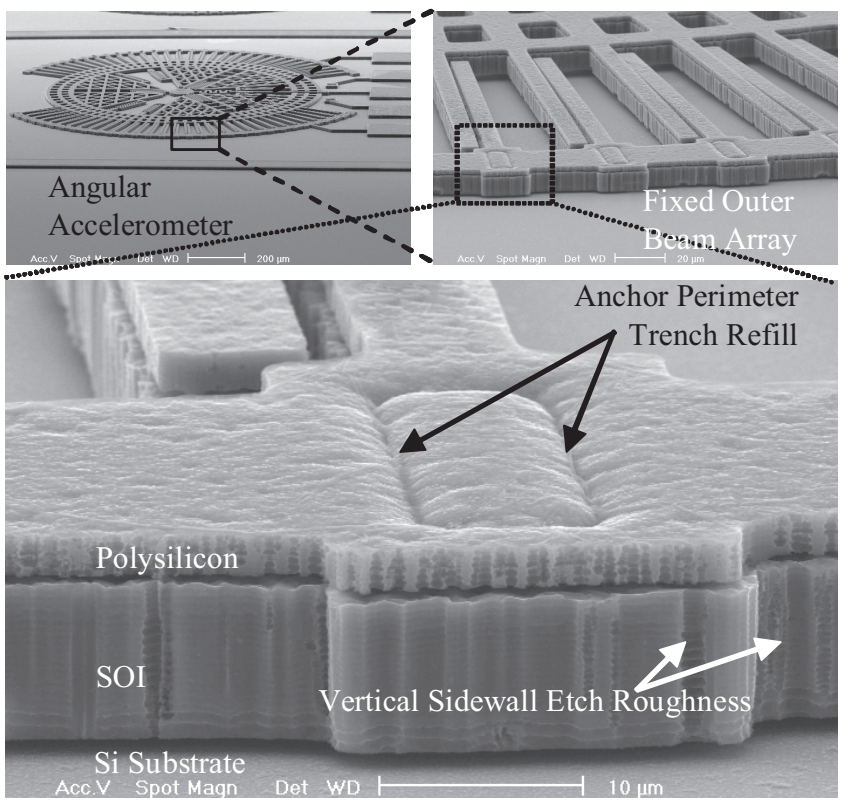

Figure 4. SOI trench refill silicon nitride encapsulated buried oxide anchor perimeter.

Low electrical resistance silicon handle wafer substrate contacts were formed using LPCVD polysilicon refilled trenches without silicon nitride sidewall passivation present. The intrinsic trench refilled polysilicon contacts were autodoped using adjacent degenerately doped SOI as a dopant source via a thermal anneal maintained at $950^{\circ} \mathrm{C}$ for 30 minutes. The test structure used to characterize the substrate contact resistance is shown in Figure 5.

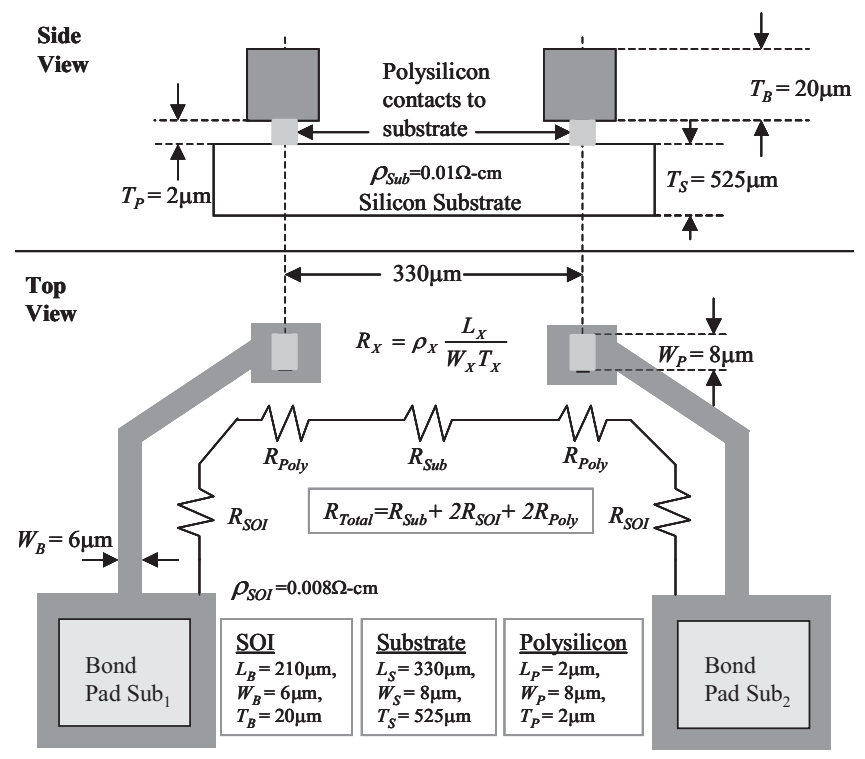

Figure 5. Polysilicon trench refill electrical substrate contact bond pad test structure schematic for angular accelerometer.

A cleaved cross section of a typical polysilicon substrate contact fabricated in $20 \mu \mathrm{m}$ thick SOI is shown in Figure 6. Electrical resistance was measured between the $S u b_{1}$ and $S u b_{2}$ bond pads via micro-manipulator probes. The pre-anneal concentration of phosphorous dopant atoms in the $20 \mu \mathrm{m}$ thick SOI film sample was $6^{*} 10^{18} \mathrm{~cm}^{-3}$. The post-anneal concentration of phosphorous dopant atoms in the polysilicon substrate contact was calculated as $1.2 * 10^{16} \mathrm{~cm}^{-3}$ based on electrical resistance measurements of $528 \Omega$ per contact [2]. This represents a polysilicon contact resistivity of $0.42 \Omega-\mathrm{cm}$. The SOI and silicon handle wafer bulk film resistivity was $0.008 \Omega-\mathrm{cm}$ and $0.01 \Omega-\mathrm{cm}$ respectively. As a result of the large difference between preanneal SOI and post-anneal polysilicon contact dopant (phosphorous) concentrations, increasing anneal temperatures and/or time can be used to provide further reduction of polysilicon contact resistance values if desired. The post thermal anneal autodoped polysilicon film can exhibit a significant stress gradient [7]. This undesirable stress gradient was minimized by removing the polysilicon film from the SOI top-side as shown in Figure 7.

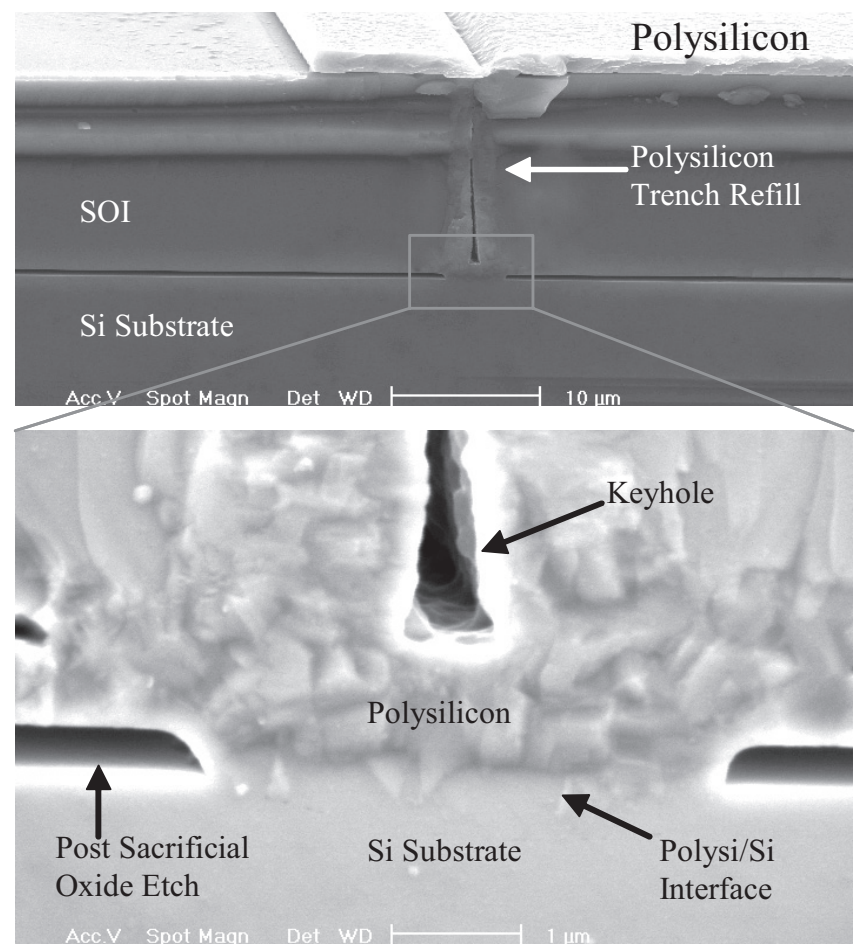

Figure 6. Cleaved cross section of autodoped polysilicon substrate contact with detail shown at single crystal silicon substrate interface (using the exposed oxide substrate contact option as described in Figure 2).

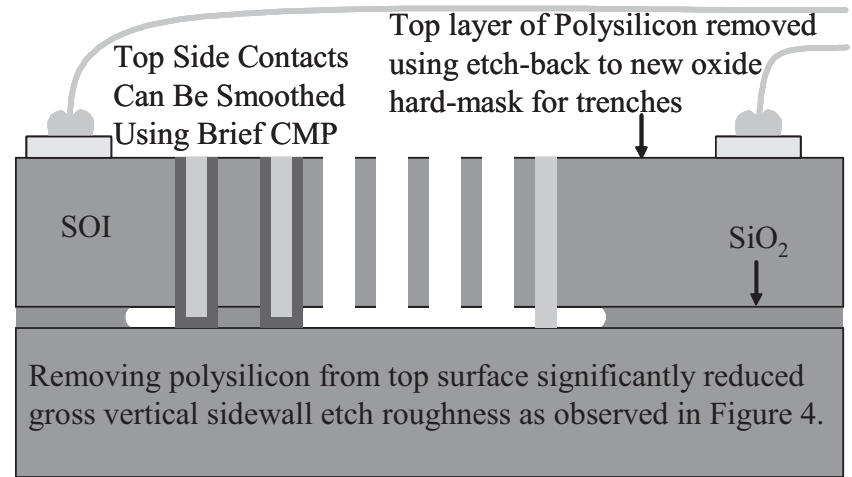

Figure 7. Second generation SOI process flow with polysilicon removed from top-side via etch-back process steps.

While the highly doped polysilicon exhibits a non-zero film stress due to thermal anneal and auto-doping effects, doped 
polysilicon is localized to small substrate contact areas isolated relatively far from the suspended sensor proof mass. Additionally, a folded beam electrical interconnect was used to reduce stress transmitted from the substrate contact towards the adjacent bond pad structure $\left(S u b_{l}\right)$ shown in Figure 8. Removing the polysilicon from the SOI top-side also eliminated the vertical sidewall etch roughness observed previously in Figure 4. Sidewall roughness was attributed to laterally etched columnar polysilicon grains shadowing the underlying SOI material.

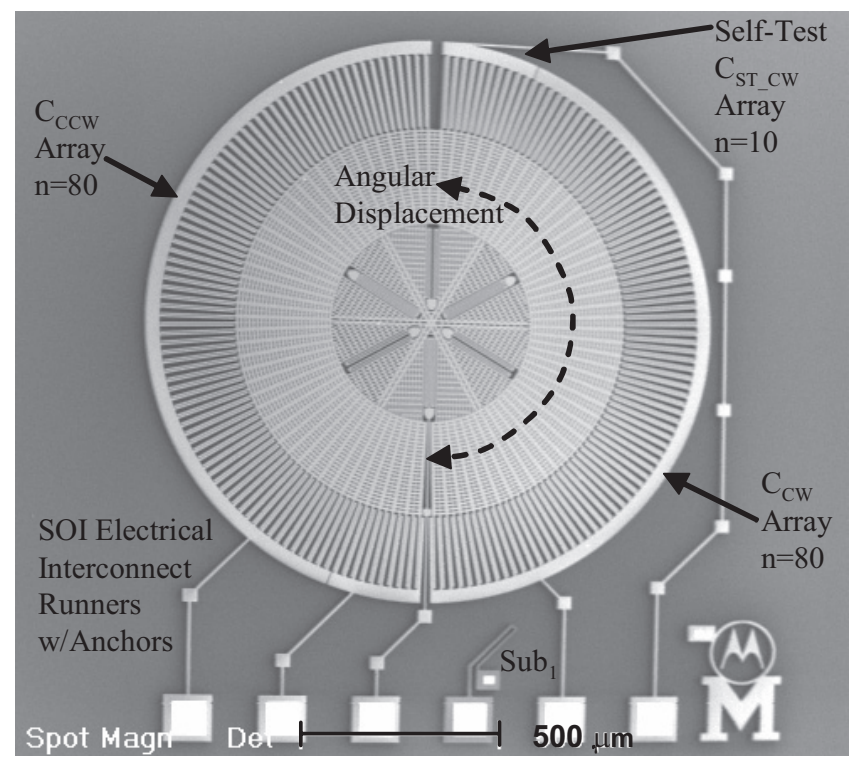

Figure 8. Angular accelerometer fabricated with the second generation SOI process flow.

\section{CHARACTERIZATION RESULTS}

Capacitive angular acceleration sensors, fabricated using the second generation SOI process, were mounted in a 16 pin ceramic dual-in-line (DIP) packages. The sensors were wire-bonded to an adjacent sensor interface application specific integrated circuit [2] (ASIC) with 2 mil diameter gold wire as shown in Figure 9.

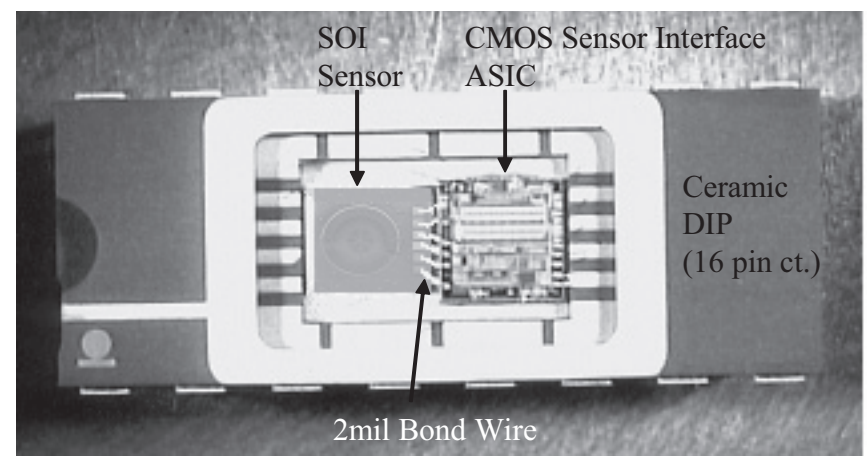

Figure 9. SOI sensor integrated with CMOS interface ASIC.

The ASIC was wire bonded to the ceramic DIP leads using 1 mil diameter gold wire. Characterization results for a sinusoidal $(2 \mathrm{~Hz})$ input angular acceleration of $40 \mathrm{r} / \mathrm{s}^{2}$ is shown in Figure 10. The CMOS ASIC output angular acceleration sensitivity was measured as $1.9 \mathrm{mV} / \mathrm{r} / \mathrm{s}^{2}$. Full scale span was calculated as $(+/-)$ $1260 \mathrm{r} / \mathrm{s}^{2}$ using output amplifier high and low rails conservatively estimated at $4.9 \mathrm{~V}$ and $0.1 \mathrm{~V}$ respectively. Angular acceleration resolution was calculated as $2.3 \mathrm{r} / \mathrm{s}^{2}$ using the measured noise and sensitivity values [2] for a typical device a shown in Figure 10.

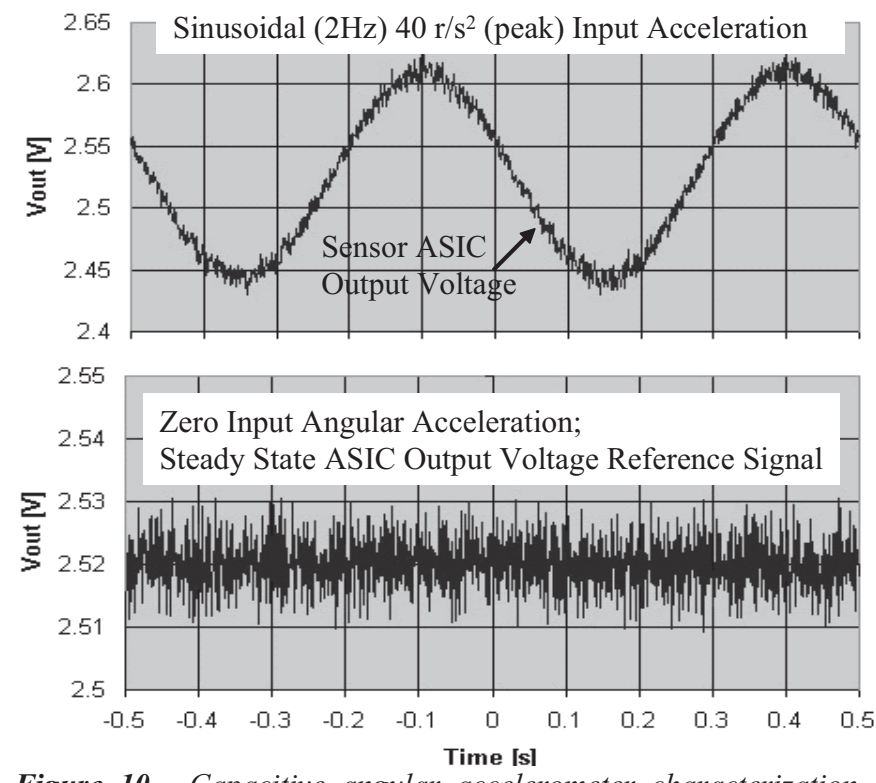

Figure 10. Capacitive angular accelerometer characterization measurements.

\section{SUBSTRATE ANCHOR SIZING}

The $\mathrm{Si}_{3} \mathrm{~N}_{4}$ passivated $\mathrm{SiO}_{2}$ substrate anchor was designed as a direct replacement of a typical timed etch $\mathrm{SiO}_{2}$ substrate anchor. As a result, minimum anchor dimensions were preserved at $20 \mu \mathrm{m}$ for both anchor schemes as shown in Figure 11.

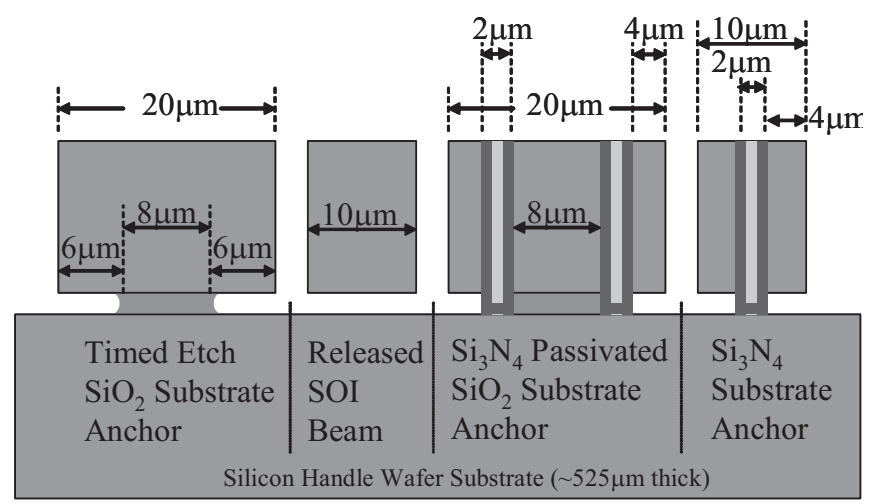

Figure 11. Comparison cross section of SOI timed etch $\mathrm{SiO}_{2}, \mathrm{Si}_{3} \mathrm{~N}_{4}$ passivated, and $\mathrm{Si}_{3} \mathrm{~N}_{4}$ substrate anchors.

The $\mathrm{SiO}_{2}$ sacrificial etch time was characterized using 6:1 aqueous HF solution (Motorola and Freescale Semiconductor proprietary buffered HF recipe) to fully release SOI beams up to $10 \mu \mathrm{m}$ in width $\left(5 \mu \mathrm{m}\right.$ lateral $\mathrm{SiO}_{2}$ undercut per side) on wafers with a $2 \mu \mathrm{m}$ thick buried oxide layer. In order to account for $\mathrm{SiO}_{2}$ etch rate variability across the device (and wafer) a target of $6 \mu \mathrm{m} \mathrm{SiO}{ }_{2}$ undercut was defined by etch time per previous buried oxide etch rate characterization experiments. A replicated angular acceleration sensor design with both Clear and Dark-Field device perimeters are shown side-by-side in Figure 12. Note that the Dark-Field device can be hermetically sealed using previously described wafer encapsulation techniques $[8,9]$. 

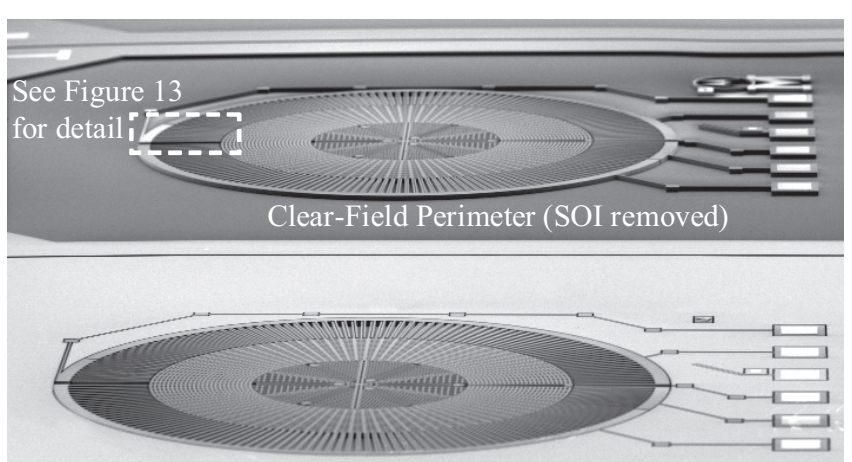

Dark-Field Perimeter (SOI intact)

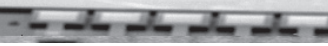

Figure 12. Clear and Dark-Field angular accelerometer designs fabricated on $20 \mu \mathrm{m}$ thick SOI wafers.

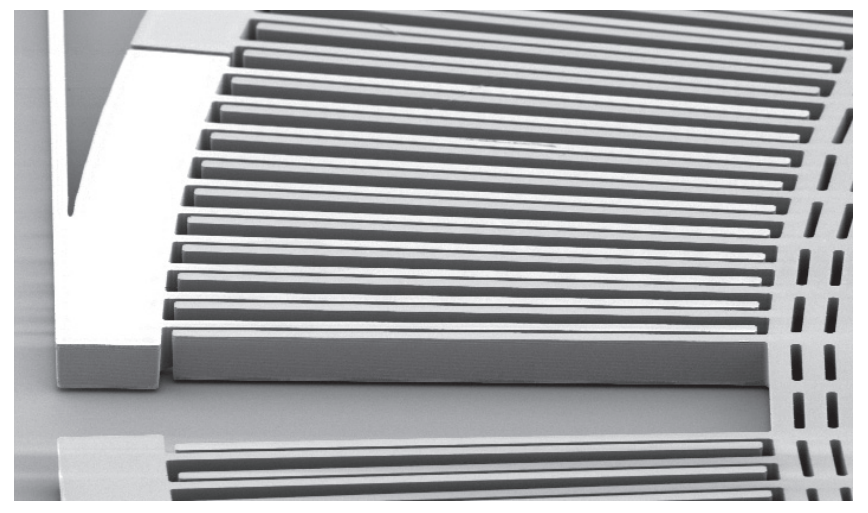

Figure 13. Clear-Field capacitive self-test array detail.

The angular accelerometer device (internal perimeter) is identical for both the Clear and Dark-Field designs. The inertial proof mass is suspended at a central hub using six folded beam springs attached to the silicon handle wafer via substrate anchors as shown in Figure 14.

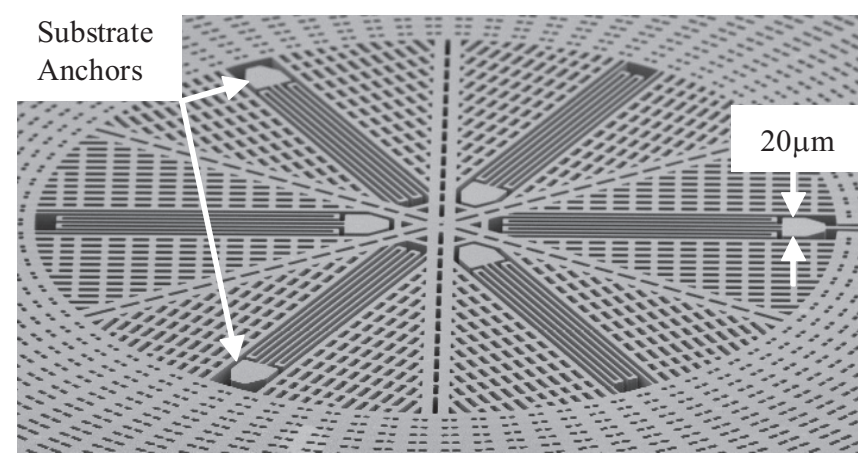

Figure 14. Capacitive angular accelerometer central hub folded beam springs with interleaved $20 \mu \mathrm{m}$ wide substrate anchors.

The $6 \mu \mathrm{m}$ lateral $\mathrm{SiO}_{2}$ etch exposes the LPCVD $\mathrm{Si}_{3} \mathrm{~N}_{4}$ passivated substrate anchors to $6: 1$ BHF for approximately $1 / 3^{\text {rd }}$ of the total etch time. Approximately $19 \%$ of the original $1.5 \mathrm{kA}$ LPCVD $\mathrm{Si}_{3} \mathrm{~N}_{4}$ film is removed during the 6:1 BHF sacrificial oxide etch step. The encapsulated $\mathrm{SiO}_{2}$ substrate anchors are protected from etchant exposure using the $\mathrm{Si}_{3} \mathrm{~N}_{4}$ film as an etch stop. Selectivity of buried oxide etch rate to silicon nitride etch rate was measured on blanket wafer tested films in excess of 100:1 using 6:1 BHF as an etchant.

Substrate anchors using only $\mathrm{Si}_{3} \mathrm{~N}_{4}$ have been previously fabricated on SOI based linear accelerometer devices [10] with minimum top and bottom anchor dimensions of $10 \mu \mathrm{m}$ and $2 \mu \mathrm{m}$ respectively as shown in Figure 11. Multiple $\mathrm{Si}_{3} \mathrm{~N}_{4}$ posts can also be applied as single trench anchors [3] limited only by the fabrication facility minimum photolithography dimension, DRIE trench width, and mask-mask maximum misalignment dimensions.

\section{CONCLUSIONS}

We have demonstrated SOI substrate anchors defined by a novel trench refill process utilizing LPCVD $\mathrm{Si}_{3} \mathrm{~N}_{4}$ as an etch stop film using standard semiconductor fabrication tools. This process was further modified with a polysilicon trench refill to provide low electrical resistance substrate contact. Characterization results of an angular acceleration sensor fabricated using this novel SOI process was also reported. Smaller lateral dimension anchors can be realized by using smaller minimum trench and buried $\mathrm{SiO}_{2}$ anchor widths. Using $1.5 \mu \mathrm{m}$ as the minimum trench width, dual trench separation, and mask-to-mask maximum misalignment error, the smallest realizable anchor is $7.5 \mu \mathrm{m}$ wide for dual trench $\mathrm{Si}_{3} \mathrm{~N}_{4}$ anchors and $4.5 \mu \mathrm{m}$ wide for single trench $\mathrm{Si}_{3} \mathrm{~N}_{4}$ anchors.

\section{REFERENCES}

[1] A. Selvakumar and K. Najafi, "High Density Vertical Comb Array Microactuators Fabricated Using a Novel Bulk/Polysilicon Trench Refill Technology," presented at IEEE Solid State Sensors and Actuators, Hilton Head, SC, pp. 138-141, 1994.

[2] G. J. O'Brien, “Design and Fabrication of MEMS Angular Rate (Gyroscope) and Angular Acceleration Sensors with CMOS Switched Capacitor Signal Conditioning," Ph.D. Dissertation, University of Michigan, Ann Arbor, MI, 2004.

[3] G. J. O'Brien and D. J. Monk, "SOI Polysilicon Trench Refill Oxide Anchor Perimeter Scheme", U.S. Patent 6,913,941, issued 2005.

[4] T. J. Brosnihan, A. P. Pisano, and R. T. Howe, "Micromachined Angular Accelerometer with Force Feedback," presented at ASME Conference and Expo, pp. 941-947, 1995.

[5] G. J. O'Brien, D. J. Monk, and K. Najafi, "Capacitive Angular Accelerometer with Dual Anchor Support," presented at IEEE Transducers, Boston, MA, pp. 1371-1374, 2003.

[6] M. A. Schmidt, "Silicon Wafer Bonding for Micromechanical Devices", presented at IEEE Solid State Sensors and Actuators Workshop, Hilton Head, SC, pp. 127-130, 1994.

[7] H. Guckel et. al, "Processing Conditions for Polysilicon Films with Tensile Strain for Large Aspect Ratio Microstructures", presented at IEEE Solid State Sensors and Actuators, Hilton Head, SC, pp. 51-56, 1988.

[8] L. J. Ristic, "Sensor Technology and Devices", Artech House Publishing, Norwood, MA, pp. 208-209, 1994

[9] R. N. Candler, W. T. Park, H. Li, G. Yama, A. Partridge, M. Lutz, and T. W. Kenny, "Single Wafer Encapsulation of MEMS Devices", IEEE Transactions on Advanced Packaging, vol. 26, No. 3, pp. 227-232, 2003.

[10] G. J. O’Brien, J. Hammond, G. Li, D. Koury, and D. J. Monk, "Outrigger; A Solid Outer Frame Lateral Accelerometer Design," presented at IEEE Transducers, Seoul, Korea, pp. 176-179, 2005. 